\title{
The Application of JDL to Suppress Sea Clutter for Shipborne HFSWR
}

\author{
Zhenyuan Ji, Chunlei Yi, Junhao Xie, and Yang Li \\ Department of Electronic Engineering, Harbin Institute of Technology, Harbin 150001, China \\ Correspondence should be addressed to Junhao Xie; xj@hit.edu.cn
}

Received 25 April 2014; Revised 27 October 2014; Accepted 28 October 2014

Academic Editor: Michelangelo Villano

Copyright (C) 2015 Zhenyuan Ji et al. This is an open access article distributed under the Creative Commons Attribution License, which permits unrestricted use, distribution, and reproduction in any medium, provided the original work is properly cited.

\begin{abstract}
This paper deals with the problem of sea clutter suppression for shipborne high frequency surface wave radar (HFSWR) based on the joint domain localized (JDL) adaptive processing algorithm. The performance of the novel method is compared with 2D FFT plus digital beamforming (FFT-DBF) and orthogonal weight in different azimuths. The results based on simulated and real data show that the novel method provides higher detection performance than others.
\end{abstract}

\section{Introduction}

HFSWR can be classified into onshore HFSWR and shipborne HFSWR according to the platform where HFSWR is located. There are many literatures about target detection and tracking based on onshore HFSWR $[1,2]$. When HFSWR is mounted on a moving shipborne platform, the first-order Bragg lines are spread in Doppler domain [3]. Besides, the sea clutter spectrum is spatially temporally coupled with each other. The background for the detection of high-velocity targets such as aircrafts and missiles is still the high-order sea clutter and noise because of their large Doppler shift. These targets can be handled just like onshore HFSWR. However, for the targets located among the spread first-order sea clutter spectrum, they are more difficult to be detected. The sea clutter suppression of space-time adaptive processing (STAP) may be better than that of the cascaded space-time processing.

STAP was proposed by Brennan and Reed in 1970s [4], which was first used in the airborne radar, to suppress ground clutter. To reduce the computational complexity of STAP, reduced-dimension STAP is used generally. For the homogeneous clutter, reduced-dimension STAP methods mainly contain JDL $[5,6]$, space-time multiple-beam (STMB) algorithm [7], linear equations [8], and the two-data-set (TDS) algorithm [9]. For the nonhomogeneous clutter, there are the direct data domain (D3) [10], AR model [11], and so on.
Among the shipborne radar clutters, sea clutter is considered to be more complex. However, the research of the sea clutter suppression for shipborne HFSWR is very limited. The orthogonal weight algorithm proposed in [12] is effective, but it is one-dimensional processing. The simulation of STAP technique has been given based on a conventional architecture of processing [13]. However, the computational complexity of the conventional STAP is rather large.

Consider a line array antenna with $N$ spatial channels and $M$ pulses per coherent processing interval (CPI). Conventional STAP algorithm uses all NM degrees of freedom (DOF). In practice, $2 N M$ independent, identically distributed (i.i.d.) secondary data samples are needed to approach the optimal performance ( $-3 \mathrm{~dB}$ loss) [14]. Because of the estimation error between real sea clutter covariance matrix in interested range cell and the estimated one using the samples around interested range bin, this method exists the signal-to-noise ratio loss depending on the number of secondary data samples to some extent. Obtaining such a large number of i.i.d. samples is difficult especially for shipborne HFSWR. Generally, if $M$ is 512 and $N$ is 7, that needs at least 7168 range data samples to estimate clutter covariance. Furthermore, even if i.i.d. samples are available, the associated computation expense makes this fully adaptive algorithm impractical. Then, a reduced-dimension STAP algorithm JDL is first used to suppress sea clutter of shipborne HFSWR in this paper. Adaptive processing is restricted to 


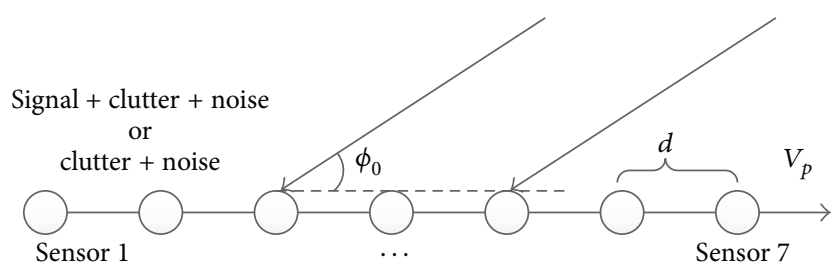

FIGURE 1: Shipborne line array.

a localised processing region (LPR) in the angle-Doppler domain for JDL, which reduces the DOF while retaining maximal gain against thermal noise [6]. The reduced DOF leads to corresponding reductions in the required samples and computation load in the conventional STAP.

This work is organized in five sections. Section 2 introduces space-time distribution of the first-order sea clutter spectrum. Section 3 discusses the theory of JDL algorithm. Section 4 introduces performance comparison and discussion. Section 5 provides the conclusion.

\section{Space-Time Distribution of the First-Order Sea Clutter Spectrum}

For the shipborne line array, the sketch map of the receiving array is depicted in Figure $1 . V_{p}$ is the velocity of a platform $(\mathrm{m} / \mathrm{s}) . \phi_{0}$ is the angle between the incident direction of radar returns and the vector of the platform velocity. $d$ is the distance between two receiving sensors $(\mathrm{m})$. The storage format of data is not one-dimensional for shipborne HFSWR, but three-dimensional. The first dimension is the data of sensors, the second dimension is the data of time-domain sweeps, and the last one is the data of range bins. In this paper, we only analyse the two-dimensional data in the range cell.

For shipborne HFSWR, platform motion causes the shift of Doppler frequency. Under ideal conditions, the space-time distribution of the first-order sea clutter spectrum in the receiving sensors can be denoted by two lines [12] as

$$
f_{d}=f_{d p} \cos \phi_{0} \pm f_{B}
$$

where $f_{d p}=2 V_{p} / \lambda, \phi_{0} \in[0, \pi], f_{B} \approx 0.102 \sqrt{f_{0}}, \lambda$ is the radar wavelength $(\mathrm{m})$, and $f_{0}$ is the carrier frequency $(\mathrm{MHz})$.

From (1), the Doppler frequency spread of the spectrum of the first-order sea clutter in the receiving sensors should be $\left[-f_{B}-f_{d p},-f_{B}+f_{d p}\right]$ and $\left[f_{B}-f_{d p}, f_{B}+f_{d p}\right]$.

\section{Joint Domain Localized (JDL) Processing}

For certain range cell, defining the sample vector in the time domain $\mathbf{x}_{n}=\left[x_{n 1} \cdots x_{n M}\right]^{T}$ for sensor $n$, the whole sample vector $(N M \times 1)$ is given by

$$
\mathbf{x}=\left[\mathbf{x}_{1}^{T}, \mathbf{x}_{2}^{T} \cdots \mathbf{x}_{N}^{T}\right]^{T} .
$$

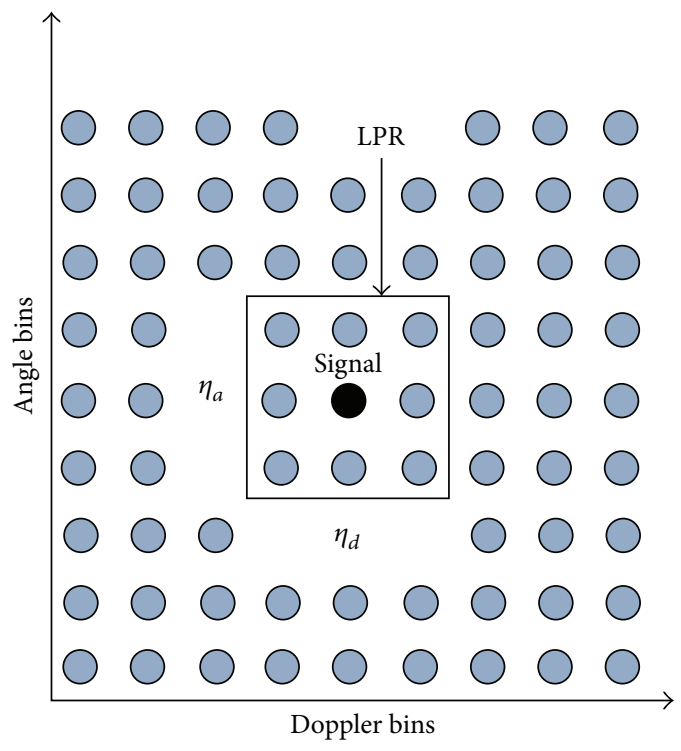

FIGURE 2: Localized processing region in JDL.

According to the definition above, the output of $\mathbf{x}$ at the azimuth $\phi_{0}$ and Doppler frequency $f_{d 0}$ can be obtained through the following equations:

$$
\begin{gathered}
x_{0}\left(\phi_{0}, f_{d 0}\right)=\mathbf{s}^{H} \mathbf{x}, \\
\mathbf{s}=\mathbf{s}_{t}\left(f_{d 0}\right) \otimes \mathbf{s}_{s}\left(\phi_{0}\right), \\
\mathbf{s}_{t}\left(f_{d 0}\right)=e^{j 2 \pi f_{d 0} T_{r}[0,1, \ldots, M-1]^{T}}, \\
\mathbf{s}_{s}\left(\phi_{0}\right)=e^{j 2 \pi(d / \lambda) \cos \left(\phi_{0}\right)[0,1, \ldots, N-1]^{T}},
\end{gathered}
$$

where $\mathbf{s}_{s}\left(\phi_{0}\right), \mathbf{s}_{t}\left(f_{d 0}\right)$ are steering vectors with azimuth $\phi_{0}$ and Doppler frequency $f_{d 0}$ in the space and time domains, respectively, $T_{r}$ is the pulse repetition period, and $\otimes$ denotes Kronecker product.

In JDL, radar signals are processed in the angle-Doppler domain. The signal vector, corresponding to the angleDoppler of interest is transformed to a single point in the angle-Doppler space. A LPR, as shown in Figure 2, is formed around the signal point and interference is suppressed in this region.

In Figure 2, the LPR covers $\eta_{a}$ angle bins and $\eta_{d}$ Doppler bins. The adaptive weights are calculated by

$$
\mathbf{w}=\mathbf{R}^{-1} \mathbf{s}_{0},
$$

where $\mathbf{R}$ is the estimated covariance matrix corresponding to the LPR of interest and $\mathbf{s}_{0}$ is the steering vector for the adaptive process.

In Figure 2, the process is finished by the following matrix:

$$
\begin{aligned}
\mathbf{T}= & {\left[\mathbf{h n} \odot \mathbf{s}_{t}\left(f_{d-1}\right), \mathbf{h n} \odot \mathbf{s}_{t}\left(f_{d 0}\right), \mathbf{h n} \odot \mathbf{s}_{t}\left(f_{d+1}\right)\right] } \\
& \otimes\left[\mathbf{s}_{s}\left(\phi_{-1}\right), \mathbf{s}_{s}\left(\phi_{0}\right), \mathbf{s}_{s}\left(\phi_{+1}\right)\right],
\end{aligned}
$$

where $\mathbf{h n}$ is Hanning window $(M \times 1)$ and $\odot$ represents the Hadamard product. 


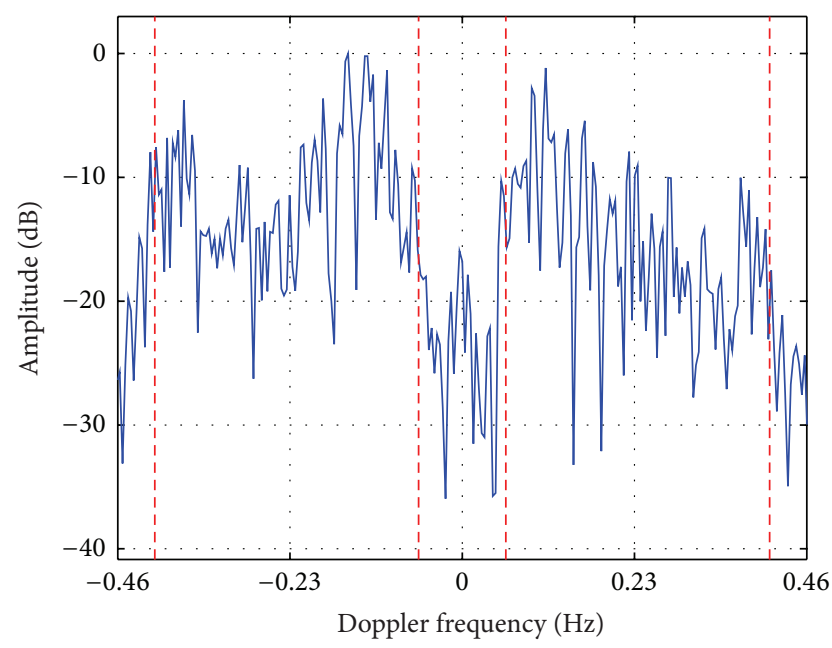

FIGURE 3: Spread spectrum of real first-order sea clutter.

So,

$$
\begin{gathered}
\mathbf{s}_{0}=\mathbf{T}^{H} \mathbf{s}, \\
\mathbf{x}_{\mathrm{LPR}}=\mathbf{T}^{H} \mathbf{x}, \\
\mathbf{R}=E\left[\mathbf{x}_{\mathrm{LPR}} \mathbf{x}_{\mathrm{LPR}}^{H}\right] .
\end{gathered}
$$

In a practical situation, $\mathbf{R}$ can be estimated by using secondary data from range cells surrounding the range cell of interest. $3^{\circ}$ spacing is chosen in the angle domain. The spacing of Doppler frequency is $0.0037 \mathrm{~Hz}$. And the adaptive weights in (4) are used to find a statistic for detection by hypothesis testing. The paper uses the modified sample matrix inversion (MSMI) statistic [6]:

$$
\rho_{\mathrm{MSMI}}=\frac{\left|\mathbf{w}^{H} \mathbf{x}_{\mathrm{LPR}}\right|^{2}}{\mathbf{w}^{H} \mathbf{s}_{0}},
$$

where $\mathbf{x}_{\mathrm{LPR}}\left(\eta_{a} \eta_{d} \times 1\right)$ is the angle-Doppler data vector from the LPR in the range cell of interest.

\section{Performance Comparison and Discussion}

In order to evaluate the performance of new method, the real data is from the shipborne radar experiment conducted from 6 to 10 September, 1998, on the Yellow Sea of China. The whole experimental system was mounted on a large barge, towed by a tugboat.

Based on the measured radar data, the spread spectrum of first-order sea clutter at 4th sensor and 17th range bin (2D FFT) is shown in Figure 3. What is more, the space-time spectrum of sea clutter at 17 th range bin (FFT-DBF) is also shown in Figure 4. The number of real data file used here is 1057. In addition, the units of the third dimension in all the figures are normalized amplitude in $\mathrm{dB}$.

In Figure 3, it is the first-order sea clutter spectrum between the two red lines. The first-order spectrum spreads obviously. In Figure 4, the space-time coupling of first-order spectrum is very strong. So the target located in Figure 4

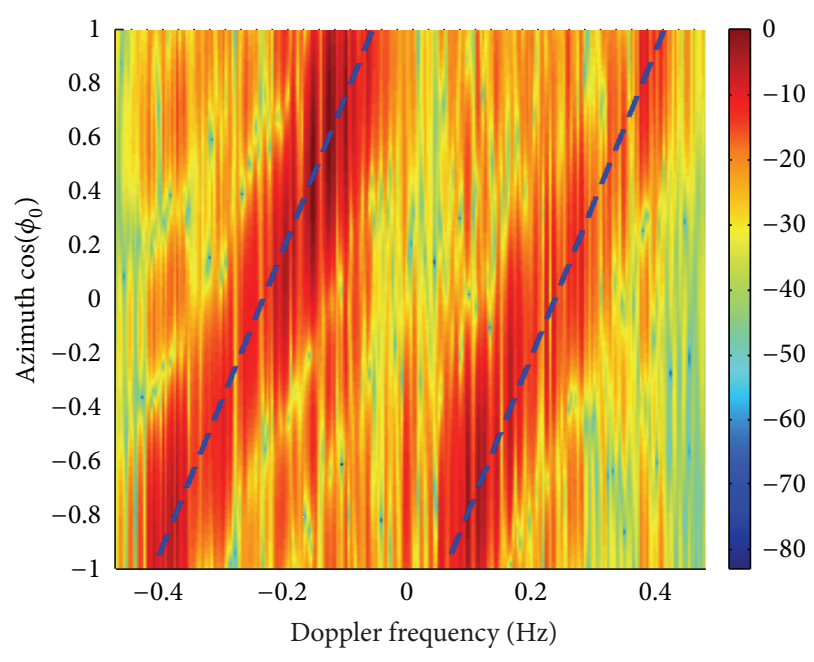

FIGURE 4: Space-time spectrum of real sea clutter. - - denotes the theoretical value of first-order sea clutter in (1).

is hard to be detected no matter only in spatial domain or frequency domain. This provides a good theoretical basis for the application of STAP algorithm.

In order to prove the truth of JDL algorithm for shipborne HFSWR, the parameters of simulated and measured data are introduced as follows. The pulse repetition interval $\left(T_{r}\right)$ is $0.26 \mathrm{~s}$, the distance of two receiving sensors $(d)$ is $14 \mathrm{~m}$, the carrier frequency $\left(f_{0}\right)$ is $5.283 \mathrm{MHz}$, bandwidth $(B)$ is $30 \mathrm{kHz}$, the number of receiving sensors $(N)$ is 7 , the number of sweeps $(M)$ is 1024 , the velocity of platform $\left(V_{p}\right)$ is $5 \mathrm{~m} / \mathrm{s}$, and the frequency resolution is $0.0037 \mathrm{~Hz}$. The azimuth of first-order sea clutter is approximately $80^{\circ}$ when Doppler frequency is $-0.2044 \mathrm{~Hz}$. The positive and negative signs mean that first-order sea clutter or targets move towards and away from shipborne HFSWR, respectively. For simulated targets, they are all added at 17 th range bin, Doppler frequencies are all $-0.2044 \mathrm{~Hz}$, and the azimuths are $95^{\circ}$ and $130^{\circ}$, respectively. Besides, the SCNR (signal to clutter plus noise ratio) of added target is $-15 \mathrm{~dB}$. It can be obtained by (8). $x_{n m}$ in (9) does not contain any targets, and the added signal matrix is also shown in (10):

$$
\begin{gathered}
\operatorname{SCNR}=20 \log _{10}\left(\frac{p \_s}{p \_c n}\right), \\
p \_c n=\frac{1}{M N} \sum_{m=1}^{M} \sum_{n=1}^{N}\left|x_{n m}\right|, \\
\operatorname{sig}=p \_s\left(\mathbf{s}_{s}\left(\phi_{0}\right) \mathbf{s}_{t}\left(f_{d 0}\right)^{T}\right),
\end{gathered}
$$

where $p_{-} s$ is the amplitude of added signal, $p_{-} c n$ is the average amplitude of clutter plus noise, and sig is added signal in the range cell of interest.

4.1. Simulated Sea Clutter Plus a Simulated Target. In this part, the performance is compared among JDL, FFT-DBF, and orthogonal weight [12]. First, the space-time spectrum 


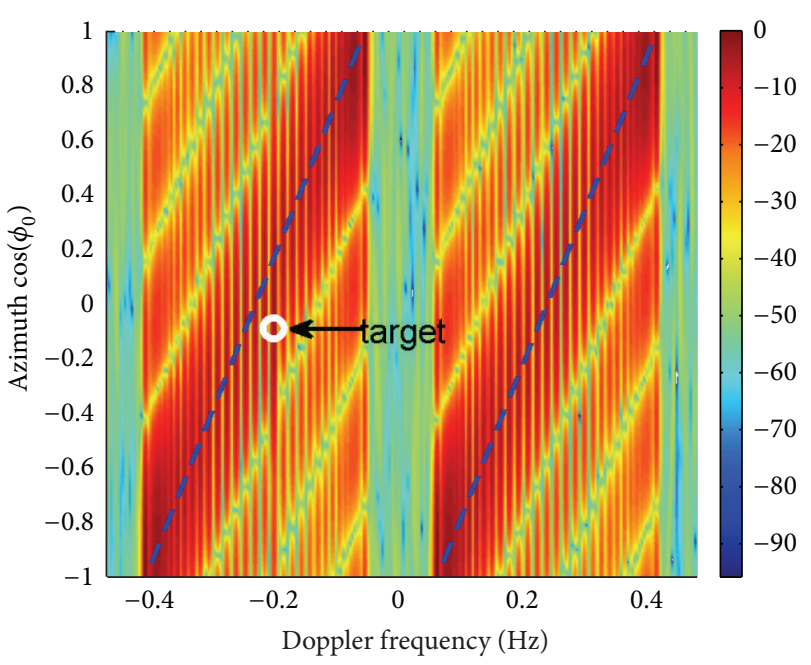

FIGURE 5: Space-time spectrum of simulated sea clutter plus a simulated target.

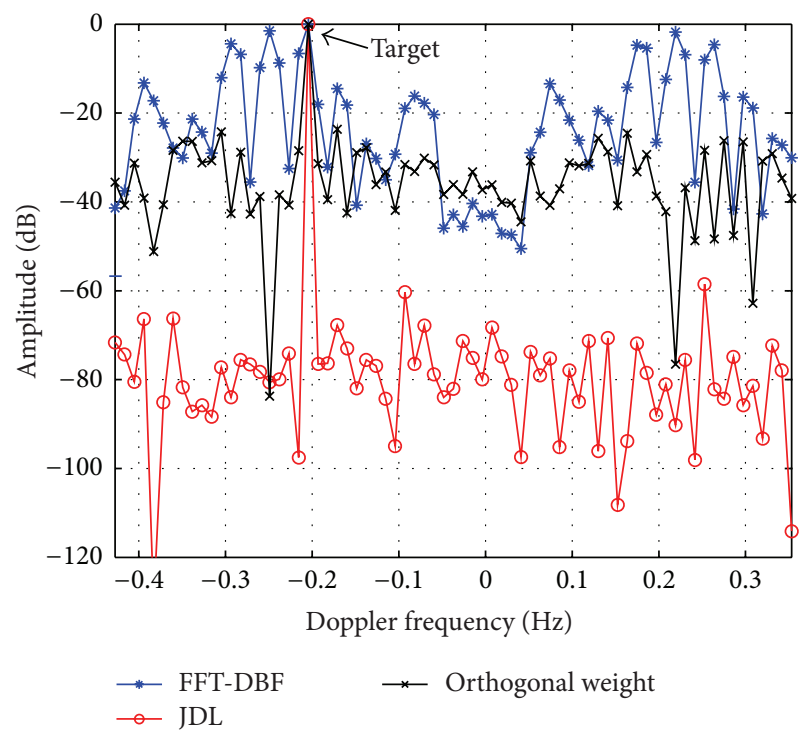

Figure 6: Compared Doppler results (simulated sea clutter, target azimuth $95^{\circ}$ ).

of simulated sea clutter with a simulated target (azimuth $95^{\circ}$ ) is shown to prove the validity of simulated data in Figure 5.

Then, the compared results are also shown in Figures 6 and 7. Because Hanning window is applied to range transform, the real target information is mainly contained in the neighbouring 3 range cells. Here, $\mathbf{R}$ is estimated using 14 secondary data cells on both sides of the range bin of interest, neglecting the first range cell on each side. The estimation methods of $\mathbf{R}$ are identical in both simulated and real data. From the two figures, FFT-DBF cannot detect the target. However, compared with orthogonal weight, the amplitude of sea clutter around the target is lower, and the first-order sea clutter is suppressed more greatly for JDL. So JDL provides the best performance of the three techniques considered.

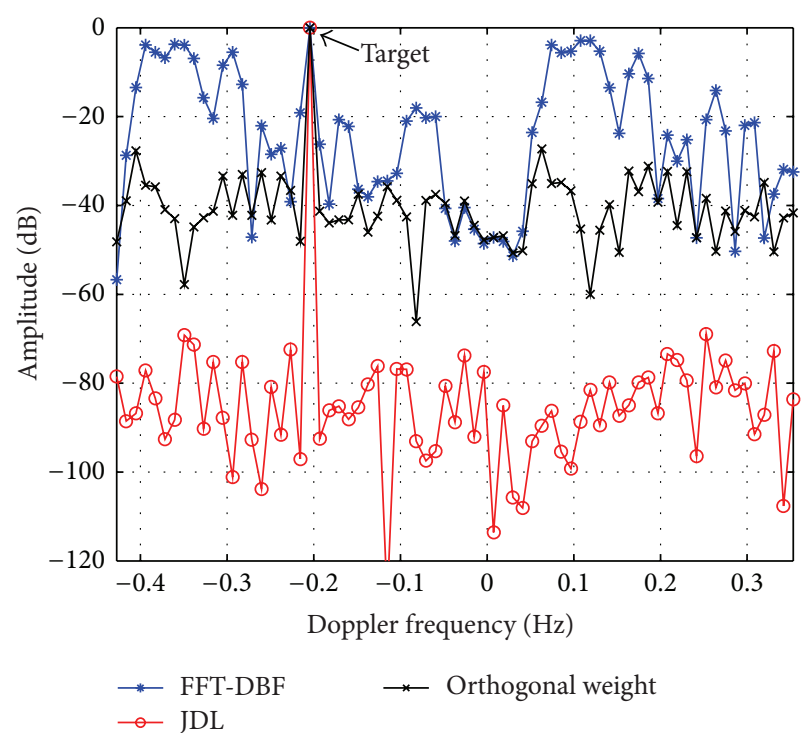

Figure 7: Compared Doppler results (simulated sea clutter, target azimuth $130^{\circ}$ ).

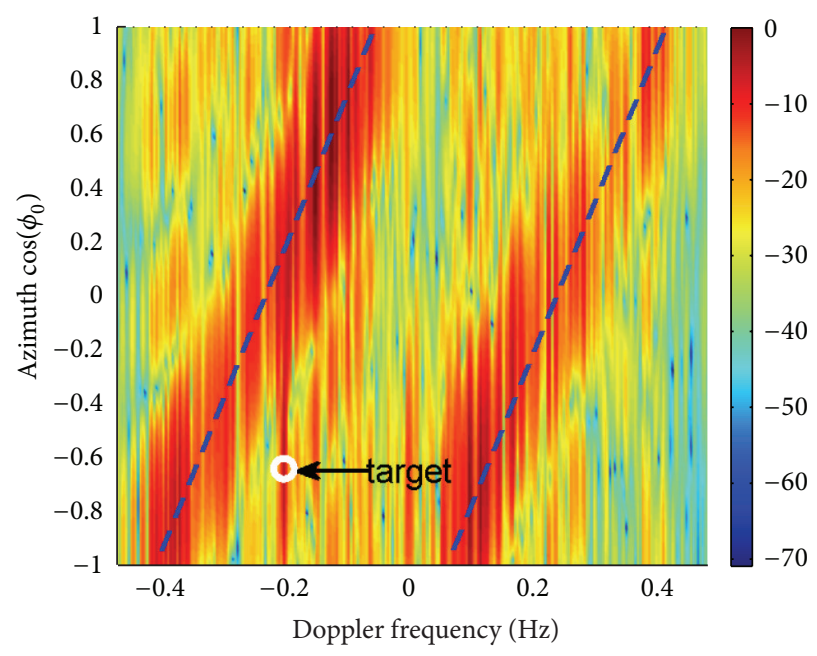

FIGURE 8: Space-time spectrum with a simulated target (real clutter data file 1057 , target azimuth $130^{\circ}$ ).

4.2. Real Sea Clutter Plus a Simulated Target. The real sea clutter data is from file 1057; then a simulated target with azimuth $130^{\circ}$ is added to the real clutter data. The position of the target is showed in Figure 8. It depicts the Doppler results in Figure 9. After that, a target with the azimuth $95^{\circ}$ is added to the real clutter data. The Doppler results of three methods are provided in Figure 10. From the figures, it is obvious that the performance of JDL is better than other methods in terms of the amplitude of sea clutter.

4.3. Measured Data. In this part, measured data is applied to illustrate the validity of JDL. The number of real data file is 1128. The Doppler frequency of the target is $-0.2044 \mathrm{~Hz}$. The azimuths of real target and the first-order sea clutter are approximately $95^{\circ}$ and $80^{\circ}$, respectively. The position of 


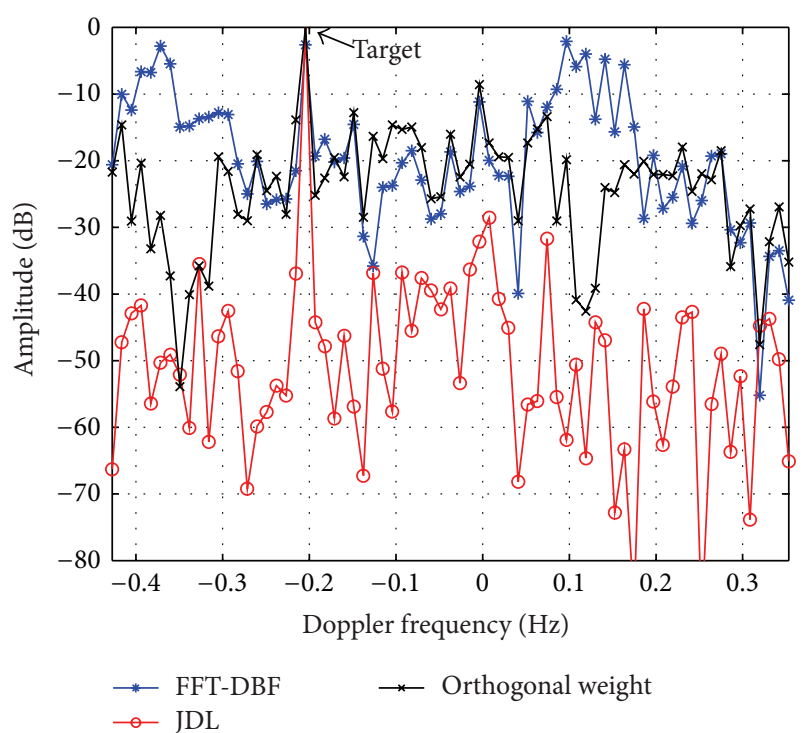

FIGURE 9: Compared Doppler results (real sea clutter, target azimuth $130^{\circ}$ ).

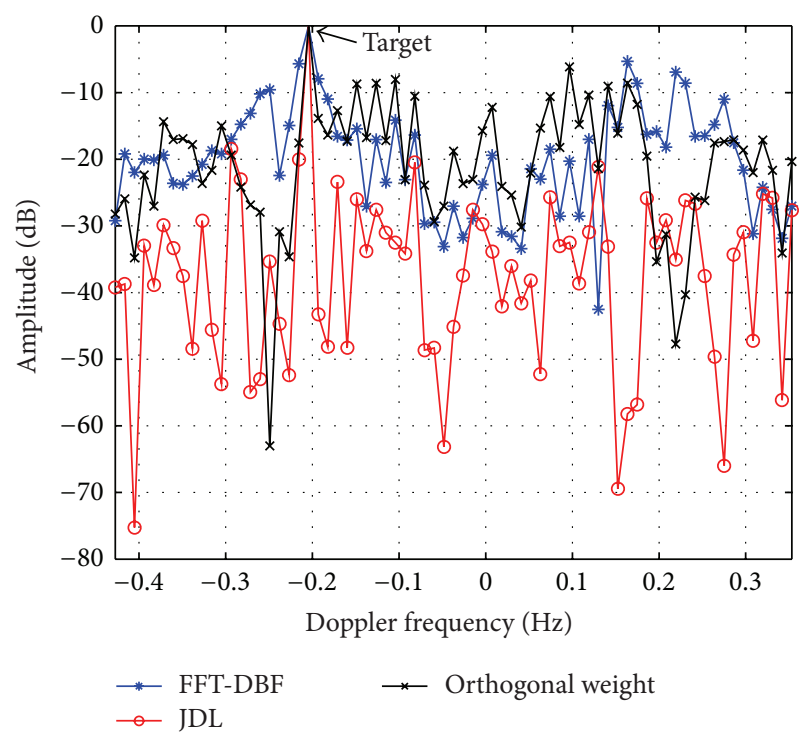

FIgURE 10: Compared Doppler results (real sea clutter, target azimuth $95^{\circ}$ ).

the target is shown in Figure 11. In azimuth $95^{\circ}$, the Doppler results of three methods are shown in Figure 12. From the figure, the SCNR of JDL is the highest, so the performance of sea clutter suppression is the best.

4.4. Performance Analysis. The performance of three methods is compared in different azimuths using simulated and real data. The conclusion can be drawn that the performance of sea clutter suppression for JDL is better than other methods from the Doppler results. This is because orthogonal weight suppresses sea clutter in one dimension, and FFT-DBF does not suppress sea clutter, but JDL suppresses sea clutter in two dimensions (STAP). Whether the performance of orthogonal

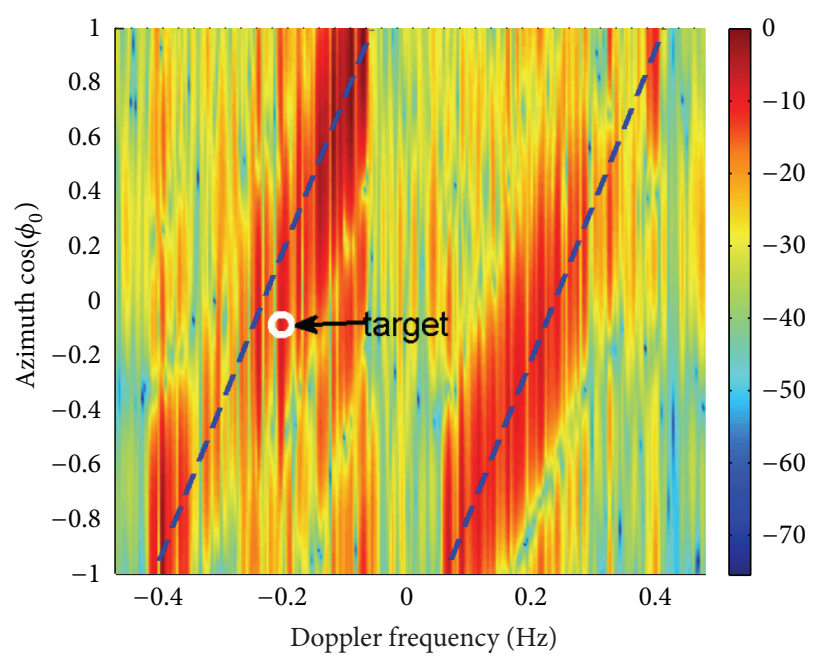

FIGURE 11: Space-time spectrum with a real target (target azimuth $\left.95^{\circ}\right)$.

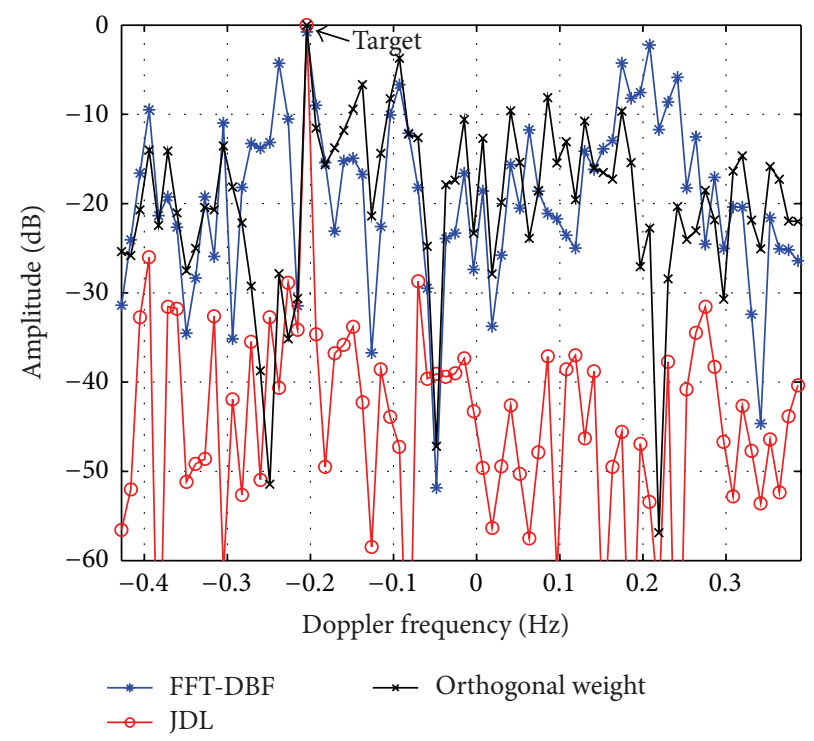

Figure 12: Compared Doppler results (target azimuth $95^{\circ}$ ).

weight outweighs that of FFT-DBF depends on the azimuth spacing between targets and the first-order sea clutter. For example, when the simulated target is far away first-order sea clutter in azimuth such as $130^{\circ}$, it can be seen that the suppression effect of orthogonal weight is better than FFT-DBF from Figures 7 and 9. However, the advantage of orthogonal weight degrades when the target is near the firstorder sea clutter in azimuth such as $95^{\circ}$ (first-order sea clutter and the target are within the same main beam) in Figures 6 and 10. This is because there is almost no loss of target gain for orthogonal weight when the azimuth of the target is far away first-order sea clutter. However, the suppression of sea clutter near the target for orthogonal weight results in loss of target gain, which is unfavourable for target detection. Because the orthogonal weight technique causes a notch in the azimuth of first-order sea clutter when the first-order sea 
clutter is lied in the same main beam with the target. The phenomenon is called main beam split. Then the main beam looks like two beams. The split in the main beam of the target leads to difficulty in azimuth estimation. But the problem for orthogonal weight can be solved by amplitude comparison of the two split beams [12].

\section{Conclusion}

In this paper, JDL algorithm is first applied to sea clutter suppression of HFSWR. The simulated and real data are used to test the performance of JDL, FFT-DBF, and orthogonal weight, which is compared in different azimuthes. Compared with FFT-DBF and orthogonal weight, the performance of sea clutter for JDL is the best. The advantage of JDL is more obvious when the target is near first-order sea clutter in azimuth. However, JDL algorithm is only available in homogenous environments [6], and its performance depends on the accuracy of estimated covariance matrix of sea clutter plus noise $(\mathbf{R})$.

\section{Conflict of Interests}

The authors declare that there is no conflict of interests regarding the publication of this paper.

\section{Acknowledgments}

This project is supported by the state key program of the Natural Science Foundation of China (Grant no. 61132005) and the National Natural Science Foundation of China (Grant no. 61102158).

\section{References}

[1] Y. Liu, "Target detection and tracking with a high frequency ground wave over-the-horizon radar," in Proceedings of the CIE International Conference of Radar Proceedings (ICR'96), pp. 2933, October 1996.

[2] R. H. Khan, "Target detection and tracking with HF radar using reciprocal SAR techniques," IEEE Aerospace and Electronic Systems Magazine, vol. 12, no. 1, pp. 40-43, 1997.

[3] J. H. Xie, Y. S. Yuan, and Y. T. Liu, "Experimental analysis of sea clutter in shipborne HFSWR," IEE Proceedings: Radar, Sonar and Navigation, vol. 148, no. 2, pp. 67-71, 2001.

[4] L. E. Brennan and L. S. Reed, "Theory of adaptive radar," IEEE Transactions on Aerospace and Electronic Systems, vol. 9, no. 2, pp. 237-252, 1973.

[5] H. Wang and L. Cai, "On adaptive spatial-temporal processing for airborne surveillance radar systems," IEEE Transactions on Aerospace and Electronic Systems, vol. 30, no. 3, pp. 660-669, 1994.

[6] R. S. Adve, T. B. Hale, and M. C. Wicks, "Practical joint domain localized processing in homogeneous and non-homogeneous environments," IEE Proceedings-Radar, Sonar and Navigation, vol. 147, no. 2, pp. 57-74, 2000.

[7] X.-K. Fan and Y. Fan, "A robust space-time multiple-beam STAP algorithm," in Proceedings of the 2nd International Conference on Signal Processing Systems (ICSPS '10), pp. V1-44-V1-47, Dalian, China, July 2010.
[8] H. Yao, "A reduced-rank STAP method based on solution of linear equations," in Proceedings of the International Conference on Computer Design and Applications (ICCDA '10), pp. 235-238, IEEE, Qinhuangdao, China, June 2010.

[9] E. Aboutanios and B. Mulgrew, "Hybrid detection approach for STAP in heterogeneous clutter," IEEE Transactions on Aerospace and Electronic Systems, vol. 46, no. 3, pp. 1021-1033, 2010.

[10] R. Dong and Z. Bao, "Direct data domain STAP algorithm for airborne radar applications," in Proceedings of the CIE International Conference on Radar Proceedings, pp. 770-772, October 2001.

[11] K.-Q. Duan, W.-C. Xie, and Y.-L. Wang, "A new STAP method for nonhomogeneous clutter environment," in Proceedings of the 2nd International Conference on Industrial Mechatronics and Automation (ICIMA '10), pp. 66-70, May 2010.

[12] J. Xie, Y. Yuan, and Y. Liu, "Suppression of sea clutter with orthogonal weighting for target detection in shipborne HFSWR," IEE Proceedings: Radar, Sonar and Navigation, vol. 149, no. 1, pp. 39-44, 2002.

[13] M. Lesturgie, "Use of STAP techniques to enhance the detection of slow targets in shipborne HFSWR," in Proceedings of the International Radar Conference, pp. 504-509, 2003.

[14] I. S. Reed, J. D. Mallett, and L. E. Brennan, "Rapid convergence rate in adaptive arrays," IEEE Transactions on Aerospace and Electronic Systems, vol. 10, no. 6, pp. 853-863, 1974. 

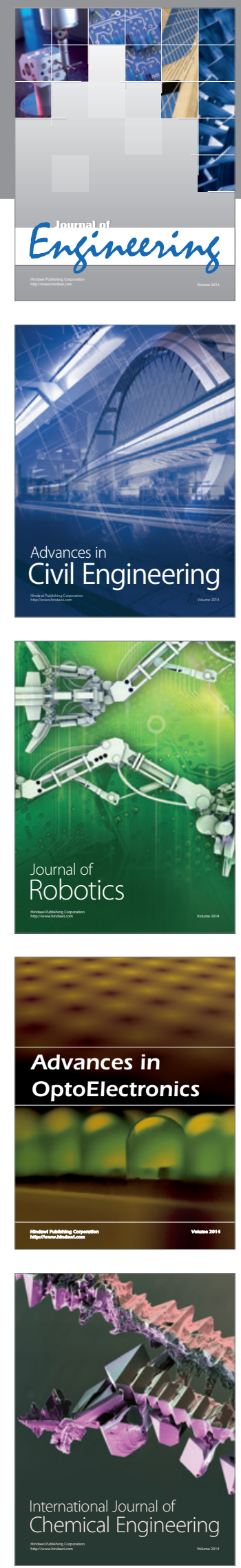

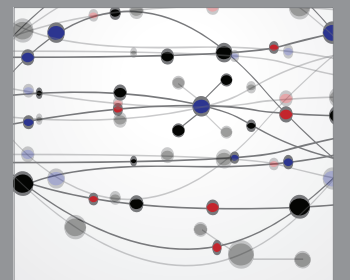

The Scientific World Journal
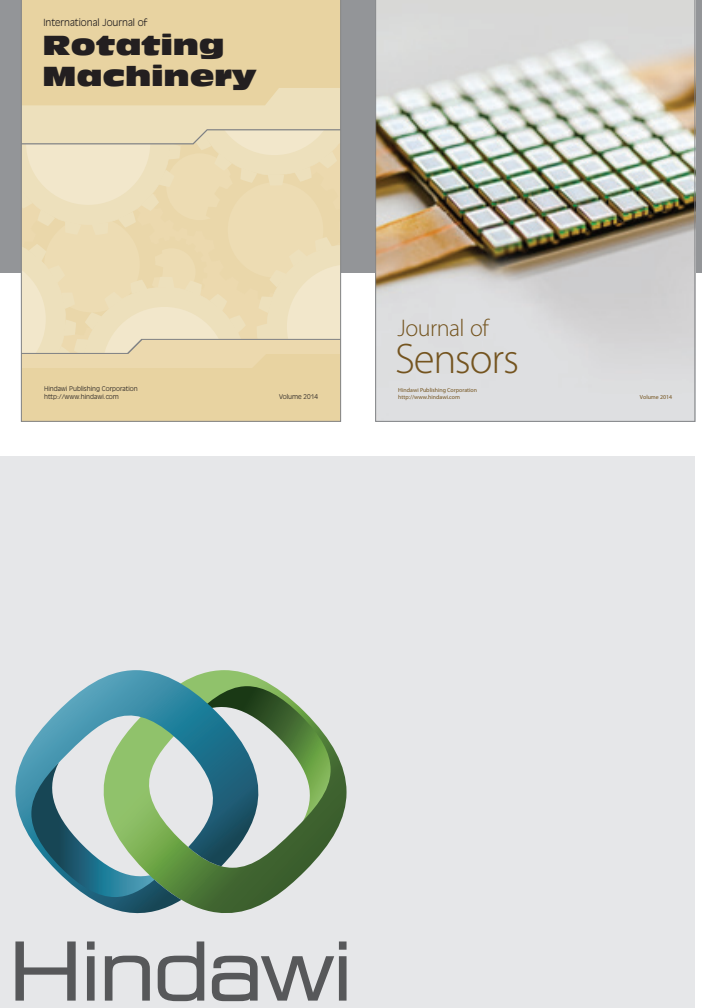

Submit your manuscripts at http://www.hindawi.com
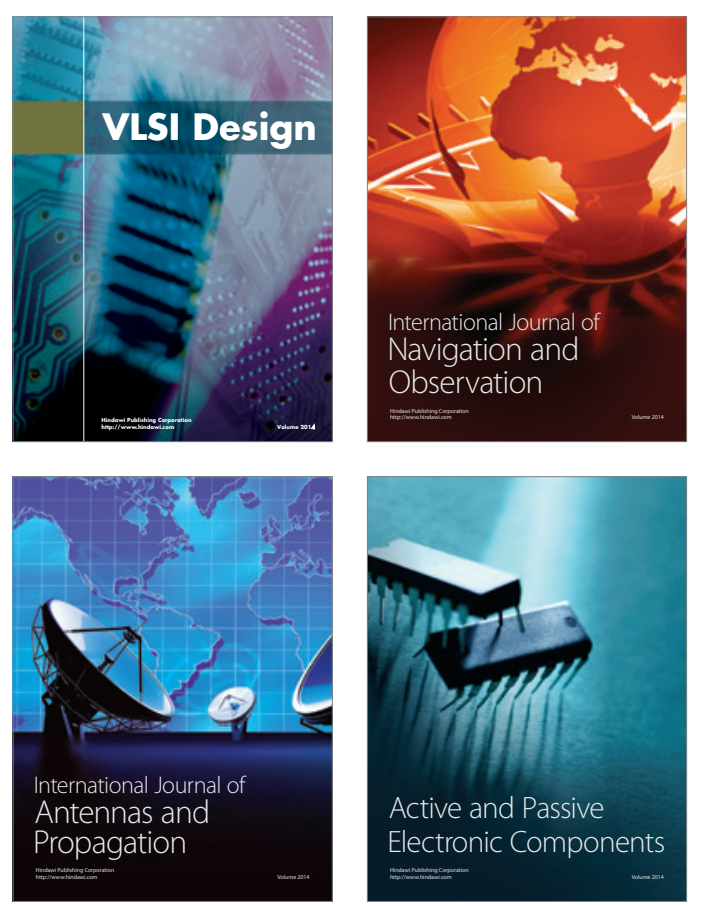
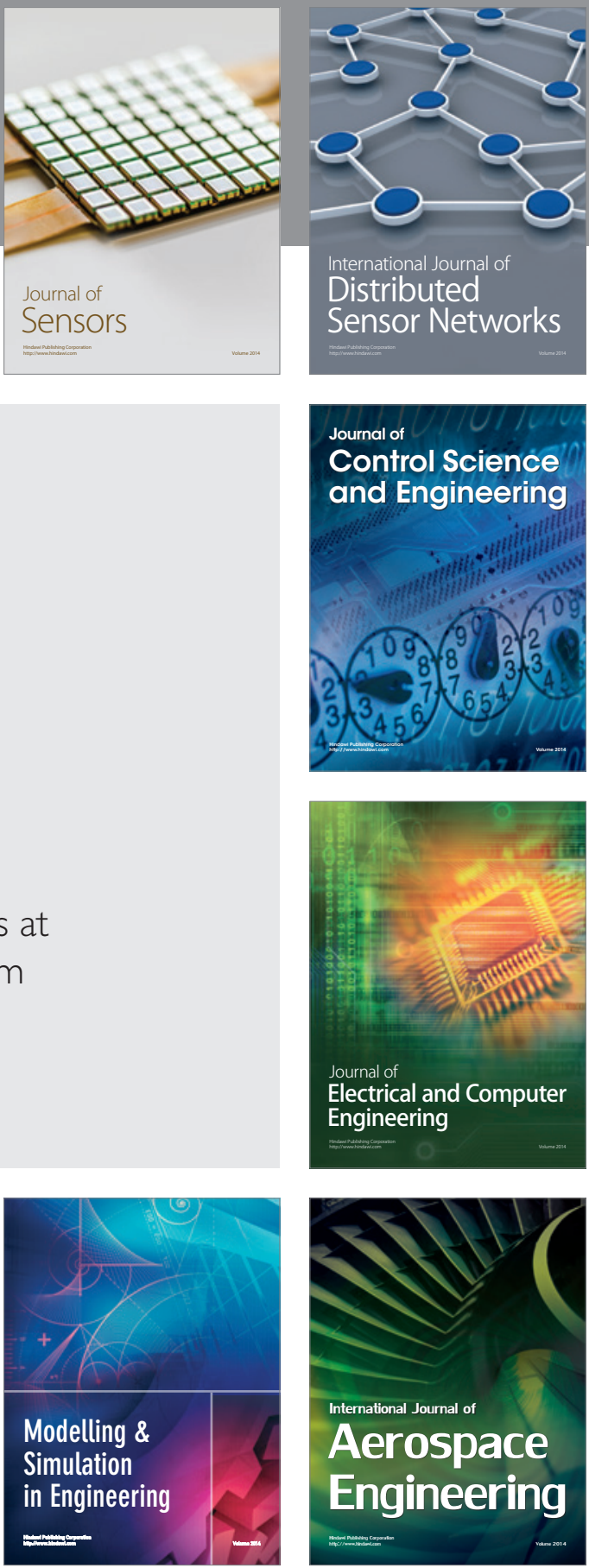

Journal of

Control Science

and Engineering
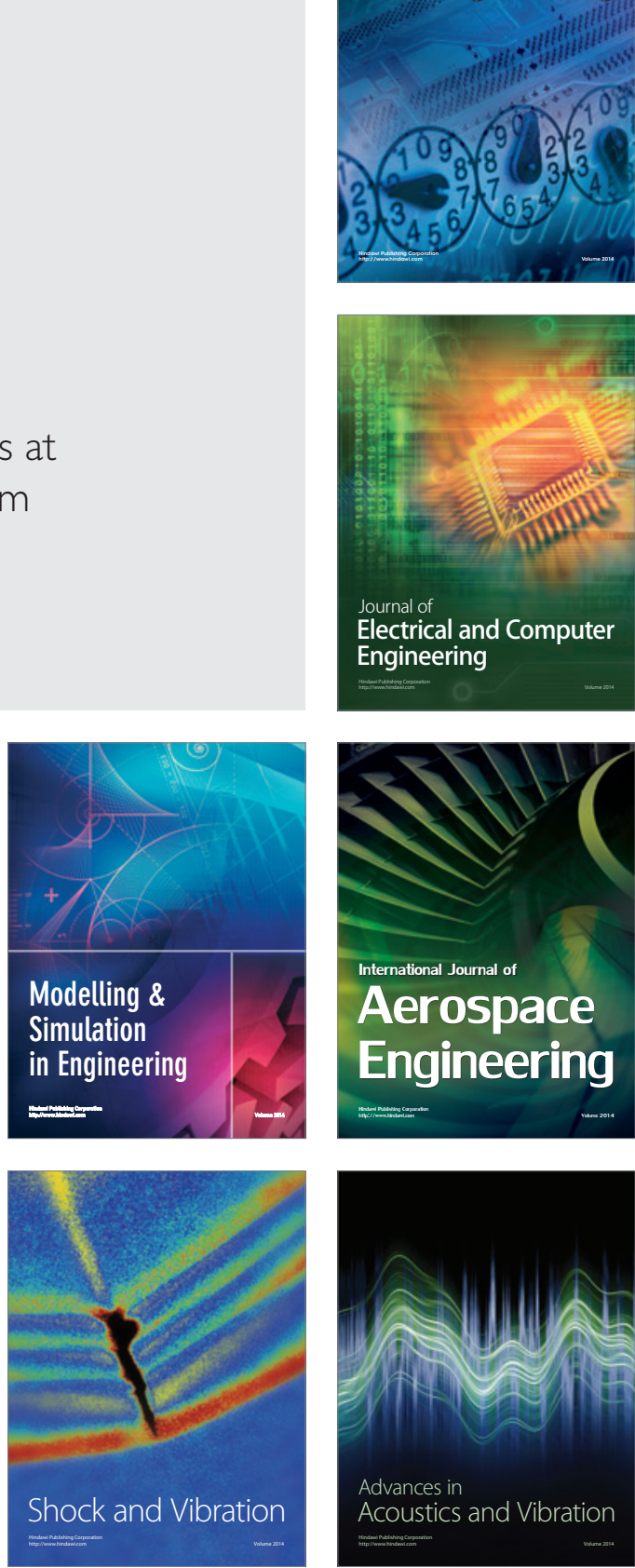